\title{
Submicron Size Patterned Nickel Soft Lithographic Masters Using Aluminium Template
}

\section{Thanigai Prakash}

Department of Medical Bionanotechnology, Chettinad Hospital and Research Institute, Kelambakkam, India. Email: thanigaiprakash@gmail.com

Received February 2 $2^{\text {nd }}, 2011$; revised March $1^{\text {st }}$, 2011; accepted March $15^{\text {th }}, 2011$.

\begin{abstract}
Nickel masters are widely used to produce surface relief grating like patterned antireflection coatings of polysiloxane material by simple embossing process. In the present work, patterned nickel master was prepared by using aluminium template, which is a foil peeled off from the commercial compact disk. Nickel was deposited using Watts nickel bath by pulsed electrodeposition for different relaxation times on patterned aluminium template. The deposition led to the transfer of patterns of aluminium replica to the nickel deposit. After deposition, the aluminium template was etched out chemically. Observation under an optical microscope showed that the obtained pattern was highly homogenous only for $T_{\text {on }}=0.1 \mathrm{~ms}, T_{\text {off }}=0.9 \mathrm{~ms}$ duty cycles as compared with other 'on' and 'off' times of pulse cycles. This simple single step cost effective nickel master can serve as stamps for the subsequent embossing process. The preparation conditions and its salient features are discussed in this paper in detail.
\end{abstract}

Keywords: Soft Lithography, Pulsed Electrodeposition, Nickel Master

\section{Introduction}

Advanced lithographic techniques for creating low dimension designs are now essential for progress in many areas of science and technology. A number of methods such as electron beam lithography, photolithography, dip pen lithography, micro contact printing, replica molding, etc $[1,2]$ are used to fabricate patterns in the submicron scale and substantial research focuses to explore new techniques with high resolution of printing and writing in the submicron regime. In particular, the established techniques like photolithography, electron beam lithography, etc., are requires specialized equipments, high capital and operational costs. But soft lithographic techniques based on embossing [3], molding [4-6], printing [7] and writing [8] have high potential to serve as cost effective and simple alternative techniques. The soft lithography processes are separated into two parts; 1) fabrication of the elastomeric elements and 2) use of these elements to pattern features in geometries defined by the element's relief structure. These two processes are typically quite different, although it is possible in some cases to use patterns generated by a stamp to produce a replica of that stamp. The structure from which the stamp is derived is known as the 'master'. It can be fabricated by any technique that is capable of producing well-defined structures of relief on a surface. Many elastomeric elements can be generated from a single master, and each such element can be used many times in patterning.

The pulsed current electrodeposition is a well established technique for deposition of metals, alloys and semiconductors by varying parameters like peak current density, 'on' and 'off' times of pulse, the sum of 'on' and 'off' times constitute one pulse cycle. The plating time was varied depending upon Ton and Toff periods, where Ton is the pulse-on-period and Toff is the relaxation period (pulse-off-period) and a duty cycle is defined as $\mathrm{T}_{\text {on }} /$ $\left(\mathrm{T}_{\text {on }}+\mathrm{T}_{\text {off }}\right)$. This method is able to reduce or completely avoid compositional gradients by regenerating the initial ion concentrations at the cathode/ solution interface at the beginning of each pulse period. Moreover, by tailoring pulse shape and duration, this technique enables close control of the nucleation and growth process and allows modification of film microstructure [9]. Also, it enables to control the thickness and chemical composition of the deposited material [10]. In this work, submicron size patterned nickel masters were prepared by pulsed current electrodeposition technique using sacrificial aluminium template and investigated its salient features. 


\section{Experimental Details}

In the present work patterned aluminium foil peeled off from a commercial non-SUPERMAS CDR (SONY) compact disc was used for transfer patterns. A compact disc is typically made of polycarbonate disk on which patterns are made by laser trilling in the form of a single spiral track. The SUPREMAS technology compact disc polycarbonate substrates are having sharp edged groves as compare with round edged groves of non-SUPREMAS. In general, the sharp edged groves lower the recording error ratio. The typical width and depth of each line in the spiral track are 0.8 and $0.5 \mu \mathrm{m}$ and separation between two such lines is about $1.6 \mu \mathrm{m}$ [11]. The diameter of a typical compact disc is much larger than the separation between two such lines and hence under an optical microscope, the lines appear parallel with nearly infinite radii of curvatures. The aluminium coating on the polycarbonate disk also has the structural patterns that appear as parallel lines under an optical microscope [12]. Both are separated independently and can be used as substrates or template to produce patterned replicas. Because of the conducting nature of aluminium foil we have employed pulsed electrodeposition technique to deposit nickel on aluminium foil. The thickness of aluminium foil is very small as compared with polycarbonate mold and so it easily rolls. In order to keep it as flat, we have stacked it with thick insulating tape on the backside. Then the aluminium foil was cleaned using methanol. LIECA 'POLYVAR 2 MET' optical microscope was used to record the images of patterned nickel masters.

The pulsed electrodeposition process was performed in room temperature using Watts nickel [13] bath $(250 \mathrm{ml}$ aqueous solution of $45 \mathrm{~g} / \mathrm{l} \mathrm{NiCl} \cdot 6 \mathrm{H}_{2} \mathrm{O}$ and $240 \mathrm{~g} / \mathrm{l} \mathrm{Ni}-$ $\mathrm{SO}_{4} \cdot 6 \mathrm{H}_{2} \mathrm{O}$ and $30 \mathrm{~g} / \mathrm{l}$ of $\mathrm{H}_{3} \mathrm{BO}_{3}$ ) at $\mathrm{pH}$ 4.5. The patterned aluminium substrate was employed as cathode and a rectangular graphite plate of similar size was employed as the anode. The graphite anode was covered with a muslin bag to avoid contamination of the bath with the graphite particles.

The nickel deposition was performed with $\mathrm{T}_{\text {off }}=0$, (b) $\mathrm{T}_{\text {on }}=0.9 \mathrm{~ms}, \mathrm{~T}_{\text {off }}=0.1 \mathrm{~ms}$ and (c) $\mathrm{T}_{\text {on }}=0.1 \mathrm{~ms}, \mathrm{~T}_{\text {off }}=0.9$ ms pulse cycles in $1000 \mathrm{~Hz}$ frequency and the current density of $5 \mathrm{~A} / \mathrm{dm}^{2}$ for one hour. Both the coatings were rinsed with deionised water, dried under a $60 \mathrm{~W}$ lamp and then subjected for chemical etching of the aluminium template at room temperature by using mixture of $1.5 \mathrm{ml}$ of sulphuric acid, $12.5 \mathrm{ml}$ of monophosphoric acid, $10 \mathrm{~g}$ of chromium trioxide and one litre of double distilled water. This etching process etches the aluminium template alone without damaging the patterned nickel costing because of its high reactivity against aluminium.

\section{Results and Discussions}

Antireflection layers are generally improves the light transmittance in solar energy tapping applications because in most of the cases solar energy receiver is a glass surface. So, refractive indices less than the glass refractive index 1.5 was required and it can be achieved by a layer with porosity or alternatively by surface relief gratings like patterned layer. Recently experimental and theoretical reports [14] proved that surface relief gratings like patterned antireflection layers are ideal for broadband applications. Because surface relief gratings are diffractive optical elements that produce zero order diffraction therefore all the higher order diffracted fields are evanescent and only the zero order propagates as in a homogenous medium. The structure and shape will define the profile of refractive index gradient here. In particular embossing polysiloxane layer using patterned nickel master within few seconds of ultraviolet curing through opposite surface of the glass substrate exhibits more than $98 \%$ percent solar transmittance. Poor adhesion of this polysiloxane material with nickel master led the structure without destruction. Generally preparation of nickel masters with submicron surface relief gratings with a scanning system like an electron beam would imply immense cost. Of course, it can be produced with holographic exposure and without subsequent process steps such as etching. Photographs of aluminium and polycarbonate mold from a small piece of commercial compact disc dismantle were shown in Figures 1(a)-(d). In order to remove the aluminium from polycarbonate mold a small piece of commercial tape was first pasted on a small compact disc piece then slowly peeled it off. Both the aluminium and polycarbonate mold are examined using optical microscope at $800 \mathrm{x}$ magnifications. The examination confirms both aluminium and polycarbonate mold have the submicron size grating like patterns, the observed optical microscopic images of aluminium and polycarbonate mold are shown in Figures 2(a) and (b) respectively.

Depositing nickel on patterned aluminium template was performed with three different trials with three different 'on' and 'off' times of pulse cycles and then the template was etched out chemically. The optical microscopic images of the patterned nickel masters prepared using three different duty cycles such as (a) $\mathrm{T}_{\text {off }}=0$, (b) $\mathrm{T}_{\text {on }}=0.9 \mathrm{~ms}, \mathrm{~T}_{\text {off }}=0.1 \mathrm{~ms}$ and (c) $\mathrm{T}_{\text {on }}=0.1 \mathrm{~ms}, \mathrm{~T}_{\text {off }}=0.9$ ms were examined at $800 \times$ magnification were shown in Figure 3. All the aluminium templates transfer the replica patterns. However the masters deposited, shown in Figure 3(c) with 'high relaxation time' $\left(\mathrm{T}_{\text {off }}\right)$ transfers the replica with high resolution as compared with 'on' and 'off' times of pulse cycles which are shown in Fig- 

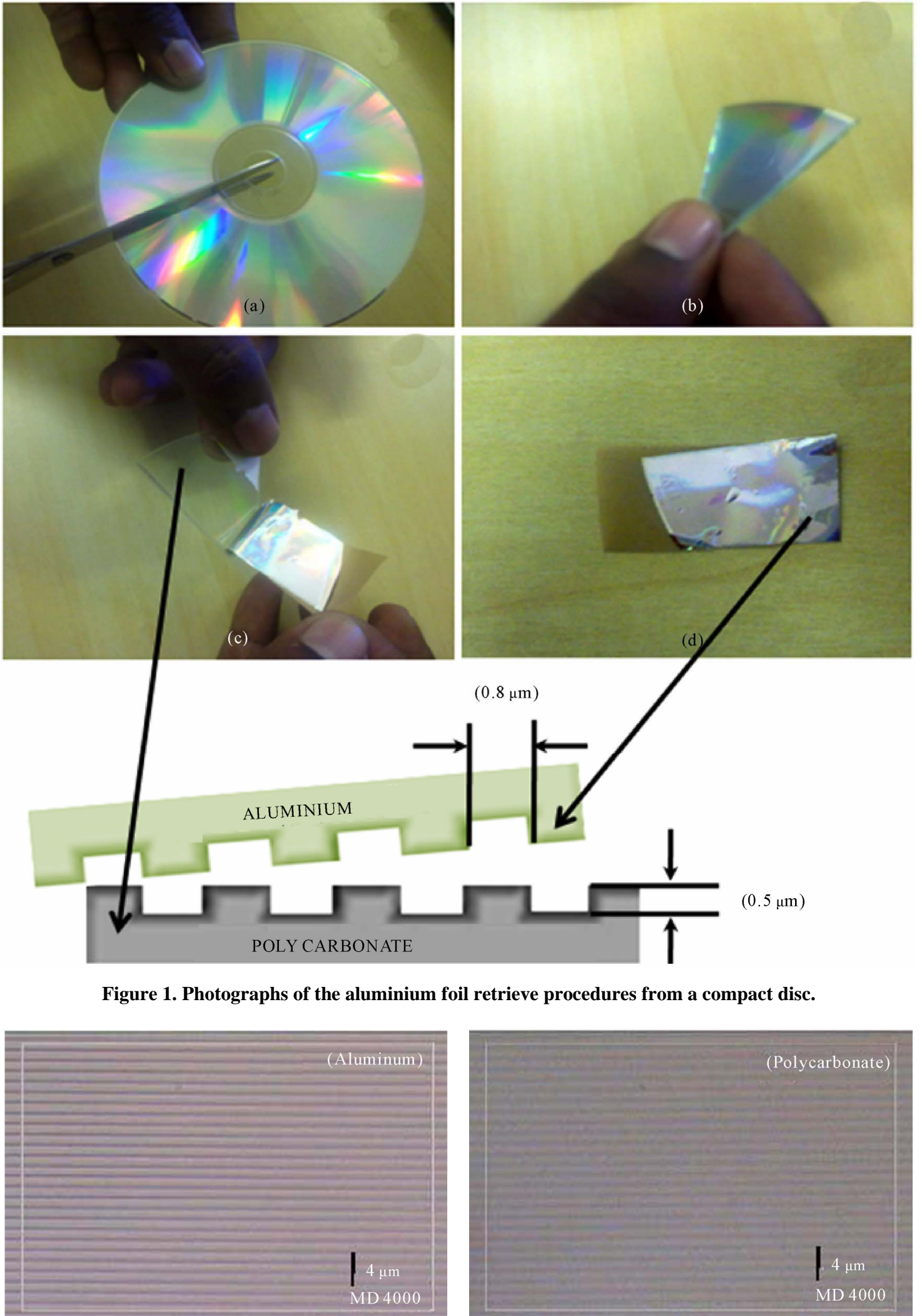

Figure 1. Photographs of the aluminium foil retrieve procedures from a compact disc.

(a)

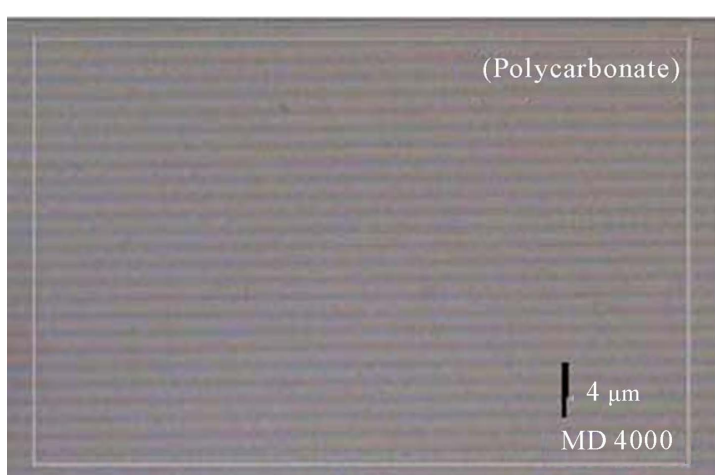

(b)

Figure 2. Optical microscopic images of the aluminium and polycarbonate templates at $\times 800$ magnification. 


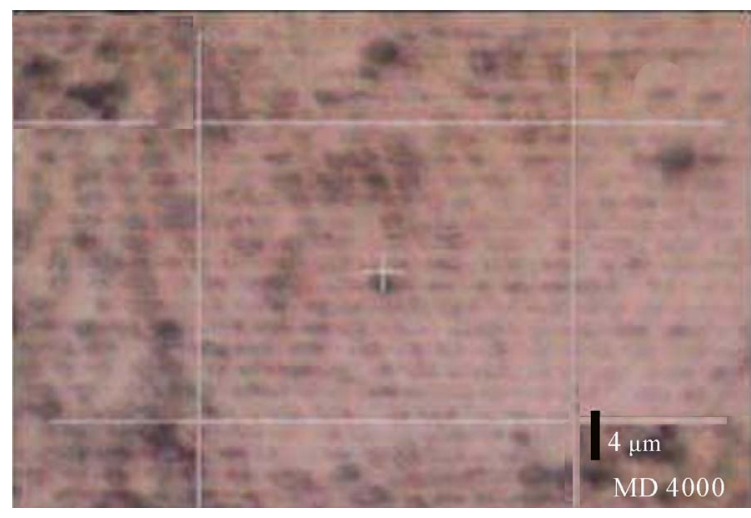

(a)

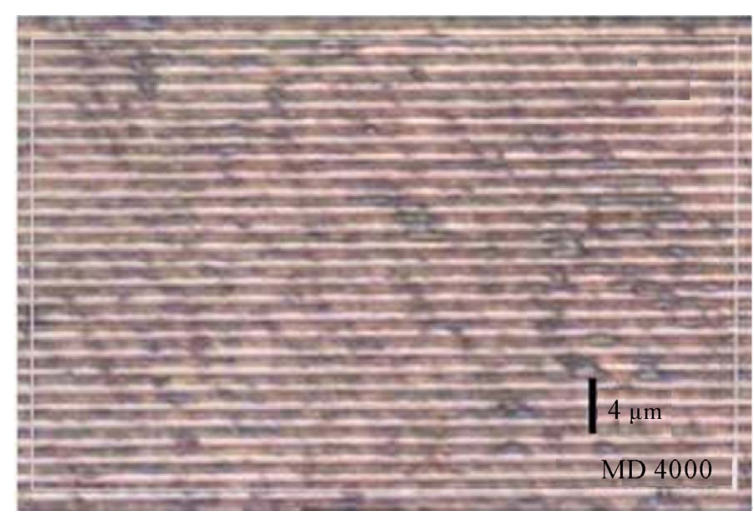

(b)

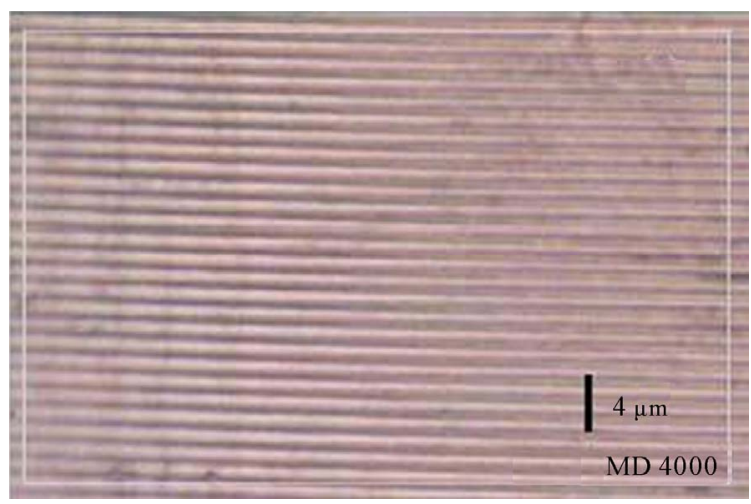

(c)

Figure 3. Optical microscopic images of the patterned nickel master in $\times 800$ magnification obtained in different duty cycles (a) $T_{\text {off }}=0$, (b) $T_{\text {on }}=0.9 \mathrm{~ms}, T_{\text {off }}=0.1 \mathrm{~ms}$ and (c) $T_{\text {on }}$ $=0.1 \mathrm{~ms}, \mathrm{~T}_{\text {off }}=0.9 \mathrm{~ms}$.

ures 3(a) and (b). The high relaxation of nickel ions interaction at cathode and the bath solution interface during deposition also increases nucleation rates leading to reduction of porosity, formation of small grains and improvements in transfer of replica pattern as compared with low relaxations. One can use these patterned nickel masters for embossing particularly to produce grating like patterned antireflection coatings of polysiloxane material.

\section{Conclusion}

In conclusion, nickel masters with $0.8 \mu \mathrm{m}$ width and 0.5 $\mu \mathrm{m}$ depth surface relief grating like pattern and separation between two adjacent lines is about $1.6 \mu \mathrm{m}$ was successfully fabricated using sacrificial aluminium template. This method can be applicable to fabricate patterned masters of other metallic and alloy materials. Also instead of electrodeposition one can use electroless deposition with the flexibility of using both polycarbonate mold and aluminium foil of compact disc as a template.

\section{REFERENCES}

[1] Y. Xia and G. M Whitesides, "Soft Lithography," Angewandte Chemie International Edition, Vol. 37, No. 5, 1998, pp. 550-575.

doi:10.1002/(SICI)1521-3773(19980316)37:5<550::AIDANIE550>3.0.CO;2-G

[2] Y. Xia and G. M. Whitesides, "Soft Lithography,” Annual Review of Materials Science, Vol. 28, 1998, pp. 153-184. doi:10.1146/annurev.matsci.28.1.153

[3] E. Kim, Y. Xia, X. M. Zhao and G. M. Whitesides, "Solvent-assisted Microcontact Molding: A convenient Method for Fabricating Three-dimensional Structures on Surfaces of Polymers,” Advanced Materials, Vol. 9, No. 8, 1997, pp. 651-654. doi:10.1002/adma.19970090814

[4] B. D. Gates and G.M. Whitesides, "Replication of Vertical Features Smaller than 2 nm by Soft Lithography,” Journal of the American Chemical Society, Vol. 125, No. 49, 2003, pp. 14986-14987. doi:10.1021/ja0367647

[5] Q. Xu, B. T. Mayers, M. Lahav, D. V. Vezenev and G. M. Whitesides, “Approaching Zero: Using Fractured Crystals in Metrology for Replica Molding," Journal of the American Chemical Society, Vol. 127, No. 3, 2005, pp. 854-855. doi:10.1021/ja043436d

[6] X. M. Zhao, Y. Xia and G. M. Whitesides, "Fabrication of Three-dimensional Micro-structures: Microtransfer molding,” Advanced Materials, Vol. 8, No. 10, 1996, pp. 837-840. doi:10.1002/adma.19960081016

[7] S. Jean, E. Menard, J. U. Park, J. Maria, M. Meitl, J. Zaumseil and J. A. Rogers, "Three-Dimensional Nanofabrication with Rubber Stamps and Conformable Photomasks," Advanced Materials, Vol. 16, No. 15, 2004, pp. 1369-1373. doi:10.1002/adma.200400593

[8] P. John Thomas, G. U. Kulkarni and C. N. R. Rao, "Dippen lithography using aqueous metal nanocrystal dispersions,” Journal of Materials Chemistry, Vol. 14, 2004, pp. 625-628. doi:10.1039/b311248a

[9] M. Emmelius, G. Pawlowski and H. W. Vollmann, "Materials for Optical Data Storage,” Angewandte Chemie International Edition in English, Vol. 28, No. 11, 1989, pp. 1445-1471. doi:10.1002/anie.198914453 
[10] D. Chowdhury, A. Paul and A. Chattopadhyay, "Patterning Design in Color at the Submicron Scale,” Nano letters, Vol. 1, No. 8, 2001, pp. 409-412. doi:10.1021/nl0155678

[11] X. Liu and G. Zangari, "High Moment FeCoNi Alloy Thin Films Fabricated by Pulsed-current Electrodeposition,” IEEE Transactions on Magnetics, Vol. 37, No. 4, 2001, pp. 1764-1766. doi: 10.1109/20.950961

[12] H. Natter, M. Schmelzer and R. Hempelmann, "Nanocrystalline Nickel and Nickel-copper Alloys: Synthesis, Characterization, and Thermal Stability,” Journal Materials Research, Vol. 13, 1998, pp. 1186-1197.
doi:10.1557/JMR.1998.0169

[13] H. S. Tung and D. J. Friedland, "An improved thermobalance for fluorination of carbon," Journal of Physics E: Scientific Instruments, Vol. 20, No. 2, 1987, p. 140. doi:10.1088/0022-3735/20/2/004

[14] A. Gombert, K. Rose, A. Heinzel, W. Horbelt, C. Zanke, B. Blasi and V. Wittwer, "Antireflective Submicrometer Surface-relief Gratings for Solar Applications,” Solar Energy Materials and Solar Cells, Vol. 54, No. 1-4, 1998, pp. 333-342. doi:10.1016/S0927-0248(98)00084-1 